\title{
GMR
}

\section{Cloning and characterization of the dehydration-responsive element-binding protein 2 A gene in Eruca vesicaria subsp sativa}

\author{
B.L. Huang ${ }^{1}$, X.K. Zhang ${ }^{2}$, Y.Y. Li ${ }^{1}$, D.Y. Li ${ }^{1}$, M.Y. Ma ${ }^{1}$, D.T. Cai ${ }^{1}$, \\ W.H. Wu ${ }^{1}$ and B.Q. Huang ${ }^{1}$ \\ ${ }^{1}$ Hubei Collaborative Innovation Center for Green Transformation of \\ Bio-Resources, College of Life Science, Hubei University, Wuhan, China \\ ${ }^{2}$ Key Laboratory of Biology and Genetic Improvement of Oil Crops, \\ Ministry of Agriculture, \\ Oil Crops Research Institute of the Chinese Academy of Agricultural Sciences, \\ Wuhan, China
}

Corresponding author: W.H. Wu

E-mail: 1305142468@qq.com

Genet. Mol. Res. 15 (3): gmr. 15038540

Received February 11, 2016

Accepted April 11, 2016

Published August 5, 2016

DOI http://dx.doi.org/10.4238/gmr. 15038540

Copyright (C) 2016 The Authors. This is an open-access article distributed under the terms of the Creative Commons Attribution ShareAlike (CC BY-SA) 4.0 License.

\begin{abstract}
Eruca vesicaria subsp sativa is one of the most tolerant Cruciferae species to drought, and dehydration-responsive elementbinding protein 2A (DREB2A) is involved in responses to salinity, heat, and particularly drought. In this study, a gene encoding EvDREB2A was cloned and characterized in E. vesicaria subsp sativa. The fulllength $E v D R E B 2 A$ cDNA sequence contained a 388-bp 5'-untranslated region (UTR), a 348-bp 3'-UTR, and a 1002-bp open reading frame that encoded 334 amino acid residues. The theoretical isoelectric point of the EvDREB2A protein was 4.80 and the molecular weight was $37.64 \mathrm{kDa}$.
\end{abstract}


The genomic sequence of $E v D R E B 2 A$ contained no introns. Analysis using SMART indicated that EvDREB2A contains a conserved AP2 domain, similar to other plant DREBs. Phylogenetic analysis revealed that EvDREB2A and DREB2As from Brassica rapa, Eutrema salsugineum, Arabidopsis thaliana, Arabidopsis lyrata, and Arachis hypogaea formed a small subgroup, which clustered with DREB2Bs from A. lyrata, A. thaliana, Camelina sativa, and $B$. rapa to form a larger subgroup. EvDREB2A is most closely related to $B$. rapa DREB2A, followed by DREB2As from E. salsugineum, A. thaliana, A. hypogaea, and A. lyrata. A quantitative real-time polymerase chain reaction indicated that EvDREB2A expression was highest in the leaves, followed by the roots and hypocotyls, and was lowest in the flower buds. EvDREB2A could be used to improve drought tolerance in crops.

Key words: Eruca vesicaria subsp sativa; Gene cloning;

Dehydration-responsive element-binding protein $2 \mathrm{~A}$

\section{INTRODUCTION}

Dehydration-responsive element-binding (DREB) proteins are transcription factors of the APETALA2/ethylene-responsive element-binding factor (AP2/ERF) family, which recognizes the dehydration-responsive element (DRE) core sequence motif (A/GCCGAC) in the promoters of stress-inducible genes (Yamaguchi-Shinozaki and Shinozaki, 1994). The DREB family is one of the most promising regulons in genetic engineering for the improvement of abiotic stress tolerance in plants (Zhao et al., 2012). Among the DREB gene family, the A-2 subgroup, including $D R E B 2 A$, is specifically involved in responses to salinity, heat, drought, and cold (Liu et al., 1998; Nakashima et al., 2000; Dubouzet et al., 2003; Sakuma et al., 2006; Qin et al., 2007). Signal transduction involves upregulation of DREB2A and the activation of various genes involved in stress tolerance in different plant species (Lata and Prasad, 2011; Mizoi et al., 2012; Morimoto et al., 2013).

Eruca vesicaria subsp sativa is one of the most tolerant Cruciferae species to drought stress (Sun and Zhang, 1999; Prakash and Bhat, 2007). Recently, we found Eruca lines that were highly tolerant to polyethylene glycol (PEG)-simulated drought stress (Huang et al., 2015). In this study, we cloned and characterized DREB2A from $E$. vesicaria subsp sativa. EvDREB2A expression profiles in different tissues of the plant were also examined.

\section{MATERIAL AND METHODS}

\section{Plant and tissue collection}

E. vesicaria subsp sativa PI 251498, which is highly tolerant to PEG-simulated drought stress (Huang et al., 2015), was used for cloning DREB2A. Seeds were germinated on filter paper immersed in liquid Murashige and Skoog medium without sugar or organic

Genetics and Molecular Research 15 (3): gmr.15038540 
components. Seven days after seed inoculation, the roots and hypocotyls of at least three plants were harvested separately and frozen immediately in liquid nitrogen, and then stored at $-80^{\circ} \mathrm{C}$. Young leaves and flower buds were collected from the field and frozen immediately in liquid nitrogen and stored at $-80^{\circ} \mathrm{C}$.

\section{Cloning full-length $E v D R E B 2 A$ cDNA and genomic DNA}

Total RNA was isolated from the different tissues using TRIzol Total RNA Extraction Reagent (TaKaRa, Dalian, China). First-strand cDNA was synthesized using 1 mg total RNA and $1 \mu \mathrm{L}$ ReverTra Ace ${ }^{\circledR}$ (100 U; Toyobo, Osaka, Japan) according to the manufacturer protocol. DREB2A amino acid sequences from various higher plant species (Brassica rapa, XP_009125600.1; Arabidopsis lyrata, XP_002871157.1; A. lyrata, EFH47416.1; Arabidopsis thaliana, AED90870.1; Arachis hypogaea, ABC60025.1; Eutrema salsugineum, AAS58438.1; Salicornia brachiate, ADE35085.1; Zea mays, NP_001105876.2; and Arundo donax, JAF93766.1) were aligned by ClustalW2 (http:// www.ebi.ac.uk/Tools/msa/clustalw2/), and highly conserved regions were retrieved. A pair of degenerated primers (DREB2A core-FP and DREB2A core-RP; Table 1) was designed to amplify a 278-bp fragment of the DREB2A coding region using cDNA from leaves and roots as templates. Two gene-specific primers (5'-RACE-DREB2A and 3'-RACE-DREB2A; Table 1) were designed for 5'- and 3'-rapid amplification of cDNA ends (RACE) to clone full-length EvDREB2A cDNA using a SMART ${ }^{\mathrm{TM}}$ RACE Amplification Kit (Clontech Laboratories Inc., Mountain View, CA, USA). Primers based on sequences of the 5'- and 3'-RACE fragments (DREB2A-FP and DREB2A-RP; Table 1) were used to amplify full-length $E v D R E B 2 A$ from cDNA and genomic DNA. All of the polymerase chain reaction (PCR) products were cloned into a pMD-18T vector (TaKaRa) and sequenced. At least five independent clones were sequenced for each PCR product to ensure accuracy.

Table 1. Polymerase chain reaction (PCR) primer sequences used in the study.

\begin{tabular}{|c|c|c|c|}
\hline PCR system & Primer name & Primer sequence $\left(5^{\prime}-3^{\prime}\right)$ & Amplicon size (bp) \\
\hline Core fragment PCR & $\begin{array}{l}\text { DREB2A-FP } \\
\text { DREB2A-RP }\end{array}$ & $\begin{array}{l}\text { TGGGAAGGAGATGGCAGTTTATGATCA } \\
\text { TACCCCAAATCCTCTGCCTAACTCC }\end{array}$ & 278 \\
\hline 5'-RACE & 5'-RACE-DREB & CCCCAAATCCTCTGCCTAACTCCCCTGAAA & 476 \\
\hline 3'-RACE & 3'-RACE-DREB & CCCAAGAAGCGGAAAGTACCAGCGAAAGGA & 1215 \\
\hline \multirow[t]{2}{*}{ cDNA PCR } & DREB cDNA FP & TAACCCAAAAGGAGAAATATCACTAG & \multirow[t]{2}{*}{1738} \\
\hline & DREB cDNA RP & ACAAAAGAAATTCGGGTCACAAGGTCAA & \\
\hline \multirow[t]{2}{*}{ Genomic DNA PCR } & DREB FP & TAACCCAAAAGGAGAAATATCACTAG & \multirow[t]{2}{*}{1738} \\
\hline & DREB RP & ACAAAAGAAATTCGGGTCACAAGGTCAA & \\
\hline \multirow[t]{3}{*}{ Quantitative real-time PCR } & $\begin{array}{l}\text { DREB-qRT-FP } \\
\text { DREB-qRT-RP }\end{array}$ & $\begin{array}{l}\text { CCCAAGAAGCGGAAAGTACCAGCGAAAG } \\
\text { CGCCCTGAAGTCCCAACCGTACA }\end{array}$ & 278 \\
\hline & actin qRT-FP & CGCCGCTTAACCCTAAGGCTAACAG & \multirow[t]{2}{*}{322} \\
\hline & actin qRT-RP & TTCTCTTTAATGTCACGGACGATTT & \\
\hline
\end{tabular}

$\mathrm{RACE}=$ rapid amplification of cDNA ends.

\section{Sequence analysis}

ORF Finder (http://www.ncbi.nlm.nih.gov/gorf/gorf.html) was used to perform open reading frame $(\mathrm{ORF})$ analysis and deduce the amino acid sequence. The organization 
of $E v D R E B 2 A$ genomic DNA was determined using the Splign software (http://www.ncbi. nlm.nih.gov/sutils/splign/splign.cgi). The theoretical isoelectric point (pI) and molecular weight $(\mathrm{Mw}$ ) were computed using the Compute $\mathrm{pI} / \mathrm{Mw}$ tool (http://web.expasy.org/ compute_pi/). Nucleotides and the derived amino acid sequences of EvDREB2A were BLAST-searched against DREB2A genes from different plant species on the National Center for Biotechnology Information (NCBI) server (http://www.ncbi.nlm.nih.gov/ BLAST). A motif scan of the deduced EvDREB2A protein was conducted using the Simple Modular Architecture Research Tool (SMART) (http://smart.embl-heidelberg.de), and the secondary structure was predicted using the SOPMA software (http://npsa-pbil. ibcp.fr/). A phylogenetic tree was constructed using the DNAMAN software (Lynnon Biosoft) based on the amino acid sequences.

\section{Expression analysis by quantitative real-time PCR}

Total RNA from leaves, roots, hypocotyls, and flower buds was extracted for quantitative real-time PCR. The primers DREB2A qRT-FP and DREB2A qRT-RP (Table 1) were used to detect $E v D R E B 2 A$ expression, and the constitutively expressed actin gene was used as an internal control to normalize $E v D R E B 2 A$ transcript levels. The quantitative real-time PCRs were performed in triplicate according to the manufacturer (Hangzhou Bioer Technology Co., Ltd., China) instructions using the comparative Ct method: the PCR amplification was performed in a reaction mix with a final $15-\mu \mathrm{L}$ volume, comprising $1.2 \mu \mathrm{L}$ cDNA, $0.2 \mu \mathrm{L}$ each gene-specific primer, $7.5 \mu \mathrm{L} 19 \mathrm{PCR}$ mix, and $5.9 \mu \mathrm{L}$ purified water. The results are reported as means $\pm \mathrm{SD}$.

\section{RESULTS}

\section{Cloning and sequence analysis of EvDREB2A}

By using degenerated primers based on the highly conserved sequence of $D R E B 2 A$ from different plant species, a 278-bp conserved fragment was amplified from $E$. vesicaria subsp sativa root, hypocotyl, leaf, and flower bud mRNA. By aligning and splicing sequences of the conserved region and the 3'- and 5'-RACE PCR products, a 1738-bp full-length DREB2A cDNA was predicted. A 1738-bp sequence was amplified from cDNA and genomic DNA using primers based on the 3'- and 5'-RACE sequences. Analysis by ORF Finder indicated that the EvDREB2A cDNA sequence contained a 388-bp 5'-untranslated region (UTR), a 1002bp ORF region that coded 334 amino acids, and a 348-bp 3'-UTR (Figure 1). The genomic $E v D R E B 2 A$ sequence did not contain introns.

\section{Analysis of the deduced amino acid sequence}

The $\mathrm{pI}$ of the EvDREB2A protein was 4.80 and the Mw was $37.64 \mathrm{kDa}$. A comparison of the amino acid sequence homologies and biochemical properties of DREB2As from the different plant species is presented in Table 2 .

Genetics and Molecular Research 15 (3): gmr.15038540 


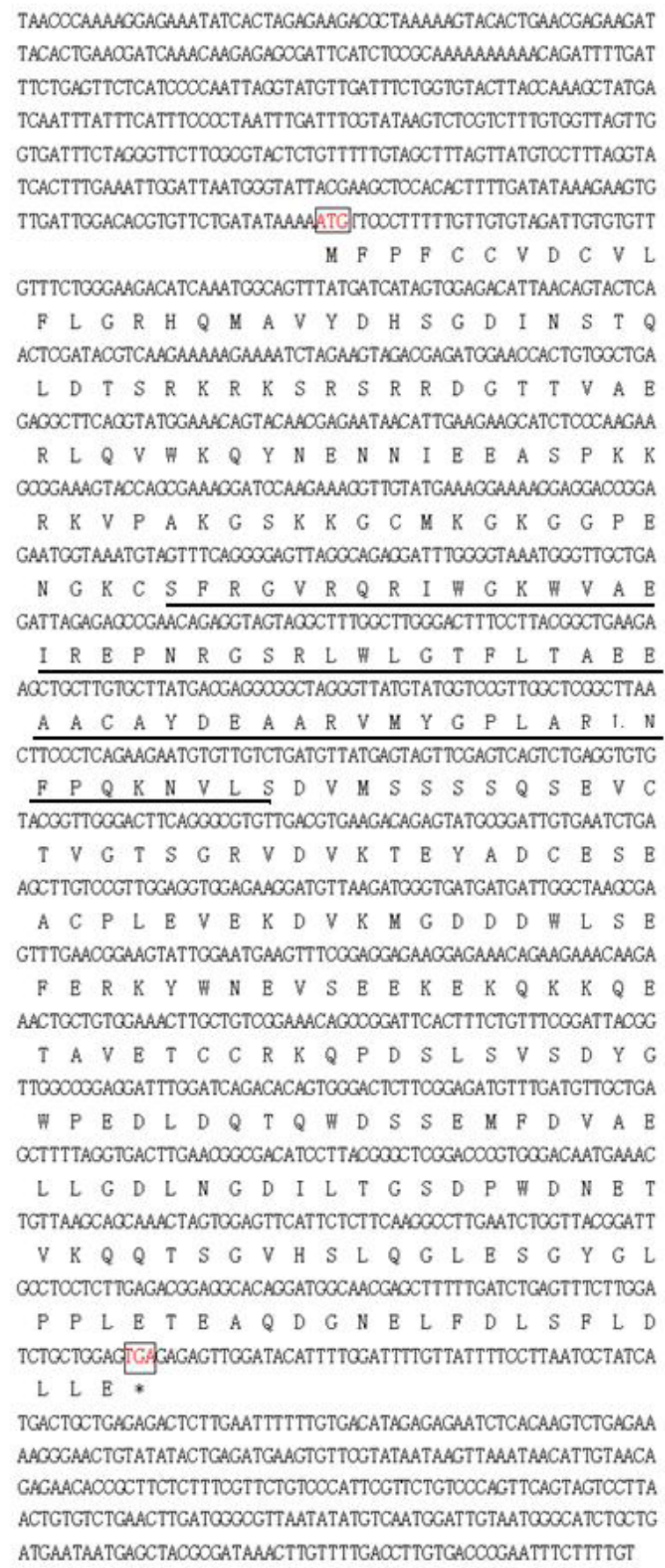

Figure 1. Full-length cDNA sequence of Eruca vesicaria subsp sativa DREB2A and the deduced amino acid sequence. The initiator and stop codons are shown in boxes. Sequences before the initiator and after the stop codons are the 5'- and 3'-UTR, respectively. The AP2 domain is underlined.

Genetics and Molecular Research 15 (3): gmr.15038540 
B.L. Huang et al.

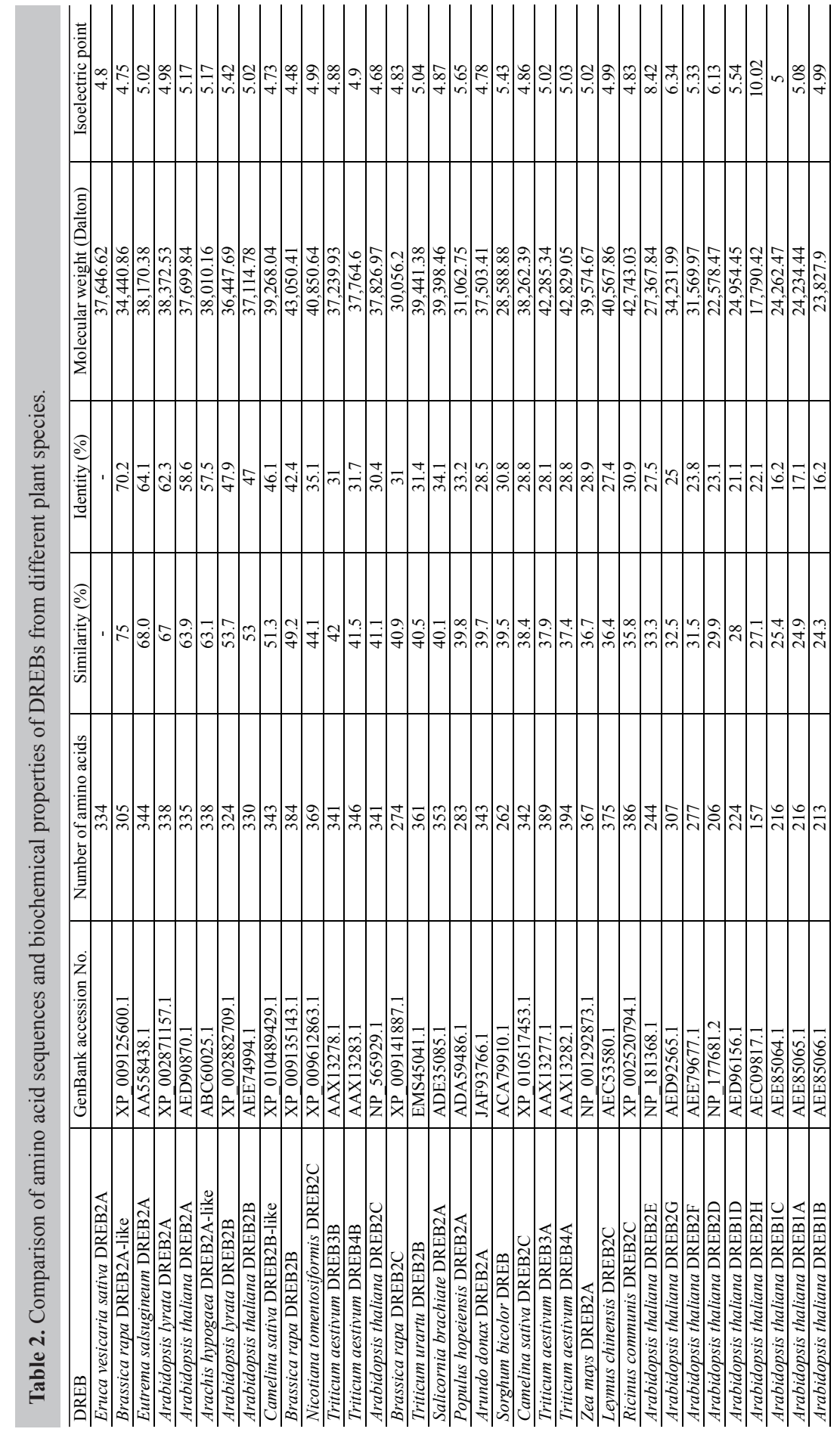

Genetics and Molecular Research 15 (3): gmr.15038540 
The BLASTp analysis revealed that the deduced EvDREB2A amino acid sequence shared the highest identity (70.2\%) with B. rapa DREB2A (GenBank accession No. XP_009125600.1), followed by $A$. lyrata DREB2A (XP_002871157.1, 62.3\% identity), A. thaliana DREB2A (58.6\% identity), and A. hypogaea DRËB2A-like (ABC60025.1, 57.5\% identity). The putative secondary structure of the deduced amino acid sequence is presented in Figure 2.

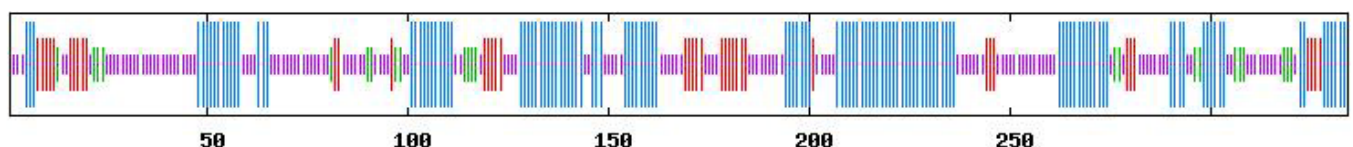

Figure 2. Secondary structure of EvDREB2A as predicted by SOPMA software. The longest lines, second longest lines, third longest lines, and shortest lines indicate alpha helices, extended strands, beta turns, and random coils, respectively.

The SMART analysis revealed that EvDREB2A contained a conserved AP2 domain (96-159aa; Figure 1). Multiple alignments of the EvDREB2A protein with other Cruciferae DREB2As are presented in Figure 3. EvDREB2A is highly similar to other Cruciferae DREB2As, and the amino sequences are identical in regions such as 74-90 and 95-129 aa (Figure 3).

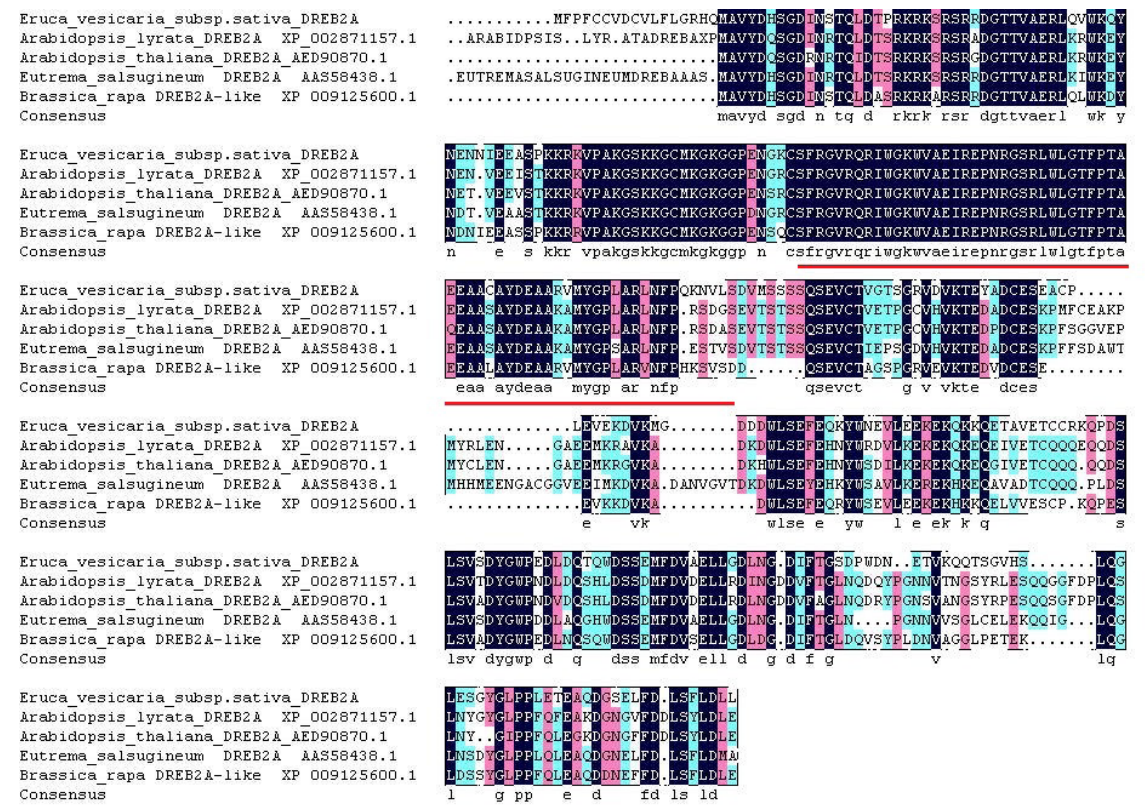

59
65
42
68
42

129
134
111
137
112

194
203
180
206
168


244
260
236
274
213

304
330
306
336
275

333
360
334
365
304

Figure 3. Multiple alignments of the N-termini of Cruciferae DREB2A proteins. The AP2 domain is underlined.

\section{Sequence alignments and phylogenetic analyses}

As shown in Figure 4, monocot DREBs clustered into one major group (M), while dicot DREBs clustered into three major groups (D1, D2, and D3). In group D1, EvDREB2A and DREB2As from B. rapa, E. salsugineum, A. thaliana, A. lyrata, and A. hypogaea formed a small subgroup, which clustered with DREB2Bs from $A$. lyrata, A. thaliana, Camelina 
sativa, and B. rapa to form a larger subgroup. EvDREB2A was most closely related to B. rapa DREB2A, followed by DREB2As from E. salsugineum, A. thaliana, A. hypogaea, and A. lyrata.

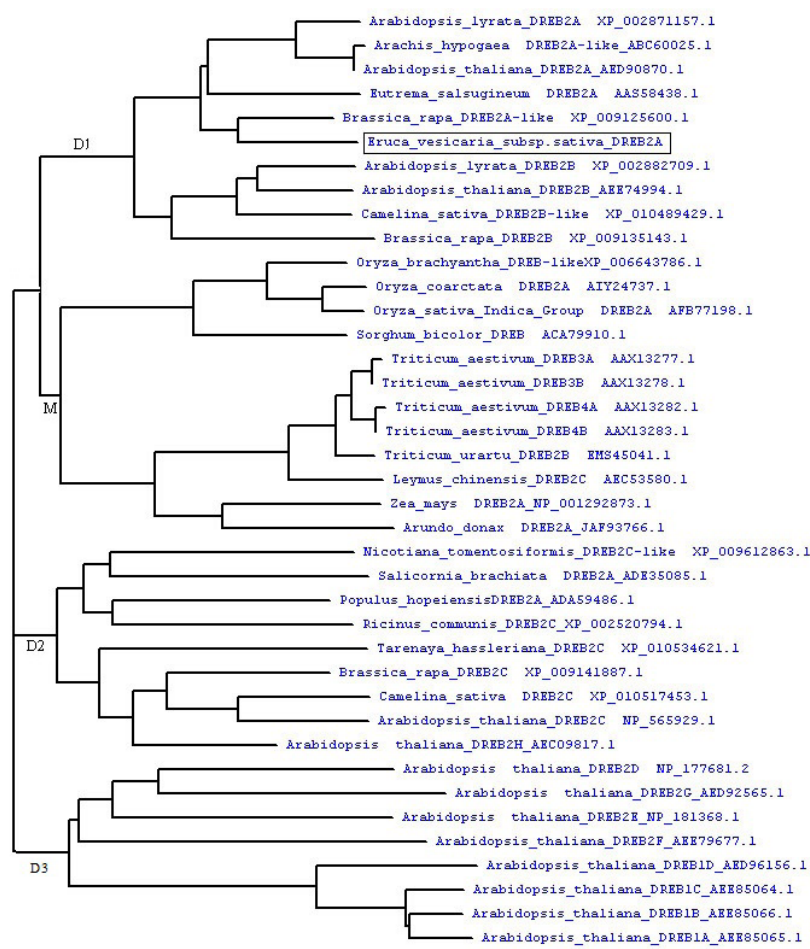

Figure 4. Phylogenetic relationships among EvDREB2A and other plant DREB proteins.

\section{Tissue-specific EvDREB2A expression}

The qRT-PCR revealed that $E v D R E B 2 A$ expression was highest in the leaves, followed by the roots and hypocotyls, and was lowest in the flower buds (Figure 5).

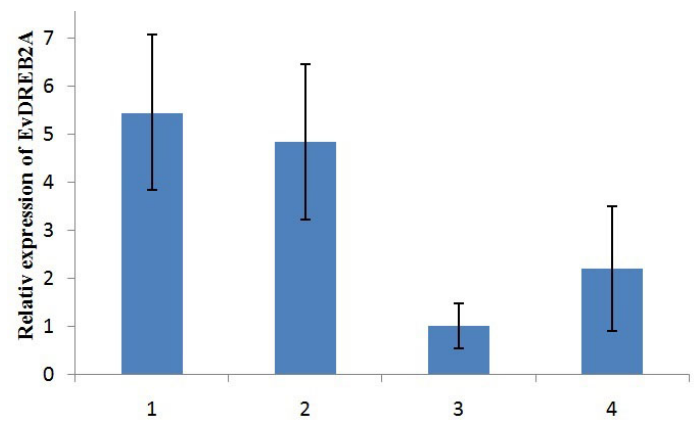

Figure 5. Relative $E v D R E B 2 A$ mRNA expression in different tissues. $E v D R E B 2 A$ expression was normalized to the amount of actin mRNA. Data are reported as means \pm SD. Column $1=$ leaf; column $2=$ root; column $3=$ flower bud; column 4 = hypocotyl.

Genetics and Molecular Research 15 (3): gmr.15038540 


\section{DISCUSSION}

In this study, we cloned the full-length $D R E B 2 A$ cDNA and genomic DNA sequences of $E$. vesicaria subsp sativa. The full-length $E v D R E B 2 A$ cDNA sequence contained a 388bp 5'-UTR, a 348-bp 3'-UTR, and a 1002-bp ORF that encoded 334 amino acid residues. The genomic EvDREB2A sequence did not contain introns, which is similar to DREB2As in A. thaliana (Liu et al., 1998) and Salicornia brachiata (Gupta et al., 2010), but different to DREB2As in rice (Dubouzet et al., 2003) and Pennisetum glaucum (Agarwal et al., 2007), which contain one intron in the genomic DREB2A sequence. The SMART analysis revealed that EvDREB2A contains a conserved AP2 domain, which is similar to that found in other plant DREBs (Sakuma et al., 2002). The phylogenetic analysis revealed that EvDREB2A and DREB2As from B. rapa, E. salsugineum, A. thaliana, A. lyrata, and A. hypogaea formed a small subgroup, which clustered with DREB2Bs from $A$. lyrata, A. thaliana, $C$. sativa, and $B$. rapa to form a larger subgroup. EvDREB2A was most closely related to $B$. rapa DREB2A, followed by DREB2As from E. salsugineum, A. thaliana, A. hypogaea, and A. lyrata.

The results of previous studies have indicated that DREB2A expression is strongly induced by dehydration, heat, and salt, and weakly induced by exogenous abscisic acid treatment (Liu et al., 1998; Nakashima et al., 2000; Sakuma et al., 2002; Gupta et al., 2010; Sadhukhan et al., 2014) and in some cases by cold (Wei et al., 2015; Chen et al., 2016). In Eucalyptus grandis, $D R E B 2 A$ is expressed in the roots, shoots, and leaves, and expression in the leaf is highest under unstressed conditions (Wei et al., 2015). In the present study, we found that EvDREB2A expression was highest in the leaves, followed by the roots and hypocotyls, and was lowest in the flower buds. EvDREB2A could be used to improve drought tolerance in crops.

\section{Conflicts of interest}

The authors declare no conflict of interest.

\section{ACKNOWLEDGMENTS}

Research supported by funds from the Science and Technology Department of Hubei Province, the Huangshi Science and Technology Bureau, the Key Laboratory of Biology and Genetic Improvement of Oil Crops, Ministry of Agriculture, China, the National Natural Science Foundation of China (\#30771382, \#30671334, \#30971807, and \#31201238), the European Commission 7th Framework Programme (ICON, 211400), and the Swedish Research Links Project.

\section{REFERENCES}

Agarwal P, Agarwal PK, Nair S, Sopory SK, et al. (2007). Stress-inducible DREB2A transcription factor from Pennisetum glaucum is a phosphoprotein and its phosphorylation negatively regulates its DNA-binding activity. Mol. Genet. Genomics 277: 189-198. http://dx.doi.org/10.1007/s00438-006-0183-z

Chen H, Liu L, Wang L, Wang S, et al. (2016). VrDREB2A, a DREB-binding transcription factor from Vigna radiata, increased drought and high-salt tolerance in transgenic Arabidopsis thaliana. J. Plant Res. 129: 263-273. http:// dx.doi.org/10.1007/s10265-015-0773-0

Dubouzet JG, Sakuma Y, Ito Y, Kasuga M, et al. (2003). OsDREB genes in rice, Oryza sativa L., encode transcription activators that function in drought-, high-salt- and cold-responsive gene expression. Plant J. 33: 751-763. http:// dx.doi.org/10.1046/j.1365-313X.2003.01661.x

Genetics and Molecular Research 15 (3): gmr.15038540 
Gupta K, Agarwal PK, Reddy MK and Jha B (2010). SbDREB2A, an A-2 type DREB transcription factor from extreme halophyte Salicornia brachiata confers abiotic stress tolerance in Escherichia coli. Plant Cell Rep. 29: 1131-1137. http://dx.doi.org/10.1007/s00299-010-0896-7

Huang B, Su J, Zhang G, Luo X, et al. (2015). Screening for Eruca genotypes tolerant to polyethylene glycol-simulated drought stress based on principal component and cluster analyses of seed germination and early seedling growth. Plant Genet. Resour. http://dx.doi.org/10.1017/S1479262115000519.

Lata C and Prasad M (2011). Role of DREBs in regulation of abiotic stress responses in plants. J. Exp. Bot. 62: 4731-4748. http://dx.doi.org/10.1093/jxb/err210

Liu Q, Kasuga M, Sakuma Y, Abe H, et al. (1998). Two transcription factors, DREB1 and DREB2, with an EREBP/AP2 DNA binding domain separate two cellular signal transduction pathways in drought- and low-temperature-responsive gene expression, respectively, in Arabidopsis. Plant Cell 10: 1391-1406. http://dx.doi.org/10.1105/tpc.10.8.1391

Mizoi J, Shinozaki K and Yamaguchi-Shinozaki K (2012). AP2/ERF family transcription factors in plant abiotic stress responses. Biochim. Biophys. Acta 1819: 86-96. http://dx.doi.org/10.1016/j.bbagrm.2011.08.004

Morimoto K, Mizoi J, Qin F, Kim J-S, et al. (2013). Stabilization of Arabidopsis DREB2A is required but not sufficient for the induction of target genes under conditions of stress. PLoS One 8: e80457. http://dx.doi.org/10.1371/journal. pone. 0080457

Nakashima K, Shinwari ZK, Sakuma Y, Seki M, et al. (2000). Organization and expression of two Arabidopsis DREB2 genes encoding DRE-binding proteins involved in dehydration- and high-salinity-responsive gene expression. Plant Mol. Biol. 42: 657-665. http://dx.doi.org/10.1023/A:1006321900483

Prakash S and Bhat SR (2007). Contribution of wild crucifers in Brassica improvement: past accomplishment and future perspectives. Proceedings of the GCIRC 12th International Rapeseed Congress, Wuhan, China, pp. 213-215.

Qin F, Kakimoto M, Sakuma Y, Maruyama K, et al. (2007). Regulation and functional analysis of ZmDREB2A in response to drought and heat stresses in Zea mays L. Plant J. 50: 54-69. http://dx.doi.org/10.1111/j.1365-313X.2007.03034.x

Sadhukhan A, Kobayashi Y, Kobayashi Y, Tokizawa M, et al. (2014). VuDREB2A, a novel DREB2-type transcription factor in the drought-tolerant legume cowpea, mediates DRE-dependent expression of stress-responsive genes and confers enhanced drought resistance in transgenic Arabidopsis. Planta 240: 645-664. http://dx.doi.org/10.1007/ $\underline{\mathrm{s} 00425-014-2111-5}$

Sakuma Y, Liu Q, Dubouzet JG, Abe H, et al. (2002). DNA-binding specificity of the ERF/AP2 domain of Arabidopsis DREBs, transcription factors involved in dehydration- and cold-inducible gene expression. Biochem. Biophys. Res. Commun. 290: 998-1009. http://dx.doi.org/10.1006/bbrc.2001.6299

Sakuma Y, Maruyama K, Osakabe Y, Qin F, et al. (2006). Functional analysis of an Arabidopsis transcription factor, DREB2A, involved in drought-responsive gene expression. Plant Cell 18: 1292-1309. http://dx.doi.org/10.1105/ tpc. 105.035881

Sun WC and Zhang T (1999). Assessment on drought tolerance of Eruca sativa genotypes from northwestern China. Proceedings of the GCIRC 10th International Rapeseed Congress, Canberra, Australia, p. 217.

Wei X, Cheng L, Ji D and Xu F (2015). The structure and expression characteristics of EgrDREB2A gene in Eucalyptus grandis. Sci. Silv. Sin 15: 80-89.

Yamaguchi-Shinozaki K and Shinozaki K (1994). A novel cis-acting element in an Arabidopsis gene is involved in responsiveness to drought, low-temperature, or high-salt stress. Plant Cell 6: 251-264. http://dx.doi.org/10.1105/ tpc.6.2.251

Zhao T, Liang D, Wang P, Liu J, et al. (2012). Genome-wide analysis and expression profiling of the DREB transcription factor gene family in Malus under abiotic stress. Mol. Genet. Genomics 287: 423-436. http://dx.doi.org/10.1007/ $\underline{\mathrm{s} 00438-012-0687-7}$

Genetics and Molecular Research 15 (3): gmr.15038540 